\title{
ENDOMETRIAL STROMAL SARCOMA - A CLINICO-PATHOLOGICAL STUDY
}

\author{
A. Sithara1, R. Vidyadhar', K. N. Sangeetha3 , K. R. Bhavadeep ${ }^{4}$, D. Sampada5, Satheesan $B^{6}$ \\ ${ }_{1}^{1}$ Assistant Professor, Department of Oncopathology, Malabar Cancer Centre, Thalassery. \\ ${ }^{2}$ Associate Professor, Department of Pathology, Kannur Medical College, Kannur. \\ 3 Professor, Department of Oncopathology, Malabar Cancer Centre, Thalassery. \\ ${ }_{4}^{4}$ Post Graduate Student, Department of Pathology, Kannur Medical College, Kannur. \\ ${ }^{5}$ Associate Professor, Department of Gynaec-Oncology, Malabar Cancer Centre, Thalassery. \\ ${ }^{6}$ Professor, Department of Surgical Oncology, Malabar Cancer Centre, Thalassery.
}

\section{ABSTRACT}

Endometrial Stromal Sarcomas (ESS) are rare tumours arising from the endometrial stroma. The present retrospective study was conducted jointly at the Malabar Cancer Centre, Thalassery and Kannur Medical College, Kannur, Kerala, between June 2011 and June 2015.

\section{AIM}

To study the age distribution, Histo-morphological features of ESS and to evaluate the prognostic factors of ESS.

\section{MATERIALS AND METHODS}

All the cases diagnosed as ESS during study period were included and other endometrial malignancies were excluded. Of the 11 cases of ESS, 10 cases were detected at Malabar Cancer Centre, Thalassery, and 1 case at Kannur Medical College, Kannur.

\section{OBSERVATIONS}

The frequency distribution of the tumour with relation to age is also evaluated. In addition to the classical morphological features, IHC for CD10 is of high value in diagnosis. ER and PR positivity may provide an avenue for hormonal therapy.

\section{RESULTS}

Significant association was observed between FIGO staging and survival of subjects. As these tumours have tendency for late recurrence, long-term follow-up is essential; i.e. once in 3 months for first year, half yearly for next 4 years and annually thereafter.

\section{CONCLUSIONS}

ESS is a rare entity and in addition to the classical morphological features, immune-staining for CD10 is of high value in its diagnosis. ER and PR positivity shows these tumours are amenable to hormonal therapy.

\section{KEYWORDS}

Endometrial Stromal Sarcoma, Undifferentiated Stromal Sarcoma, Mitotic Index, Lymphovascular Invasion, Prognosis.

HOW TO CITE THIS ARTICLE: Sithara A, Vidyadhar R, Sangeetha KN, et al. Endometrial stromal sarcoma - a clinico-pathological study. J. Evolution Med. Dent. Sci. 016;5(46):2922-2928, DOI: 10.14260/jemds/2016/680

\section{INTRODUCTION}

Endometrial Stromal Sarcomas (ESS) are rare, malignant neoplasms arising from the endometrial stroma constituting about 1-3\% of gynaecological malignancies. Carcino-sarcomas and leiomyosarcomas constitute $85 \%$ of uterine sarcomas and ESS constitute the rest (15\%).[1] The cells in ESS have resemblance to the normal endometrial stromal cells. They generally occur in younger patients than Carcino-sarcomas with a peak incidence in the $4^{\text {th }}$ and $5^{\text {th }}$ decades; 10 to $25 \%$ of affected women are premenopausal. ESS is an indolent tumour with local recurrences and distant metastasis occuring even 20 years after initial diagnosis.[2] According to the terminology first proposed by Norris and Taylor in 1966, separation into low- and high-grade categories was solely based upon mitotic activity; if a tumour which morphologically resembles

Financial or Other, Competing Interest: None.

Submission 23-04-2016, Peer Review 18-05-2016,

Acceptance 24-05-2016, Published 09-06-2016.

Corresponding Author:

Dr. R. Vidyadhar

Department of Pathology,

Kannur Medical College and Hospital,

Anjarakandy $(P)$,

Kannur-670612,

Kerala, India.

E-mail:vrrmmc_doc@yahoo.co.in

DOI: $10.14260 /$ jemds/2016/680 endometrial stroma had less than 10 mitoses/10 High Power Field (HPF), it was considered low-grade, whereas a tumour with greater than 10 mitoses/10 HPF was considered high grade.

In the current WHO classification scheme, there remain two categories of endometrial sarcomas, low grade and undifferentiated, which is based upon differences in tumour morphology rather than mitotic activity. Stromal nodule is a well-circumscribed neoplasm usually $<10 \mathrm{~cm}$ in size on histological examination. They are composed of endometrial stromal cells arranged around numerous vessels resembling spiral arterioles with well-demarcated interface between the tumour and the surrounding myometrium with only slight interdigitations between the two, but this should not exceed 3 $\mathrm{mm}$ in depth. Low grade ESS appears grossly as an intramyometrial, polypoidal or diffusely infiltrative mass. Histologically, they consist of well-differentiated endometrial stromal cells with a plexiform capillary network, which may invade the myometrium in a tongue-like fashion and may involve lymphatic channels (Hence, the old terminology endolymphatic stromal myosis).

Mitotic activity confers little prognostic value. Stage I tumours with minimal cytological atypia and low mitotic index have high relapse rates. This emphasizes the difficulty in assigning recurrence risk in stromal sarcomas based on the degree of differentiation, atypia and mitotic activity. Low 
grade ESS may exhibit other forms of differentiation including smooth muscle, epithelioid, glandular and sex-cord differentiation. At the molecular genetic level, most cases of low-grade ESS are characterized by reciprocal chromosomal translocation, $\mathrm{t}(7 ; 17)$, (p15; q21), which generates a JAZF1JJAZ1 fusion gene product. Stromal nodules have the same genetic aberration. The prognosis for low-grade endometrial stromal tumours is guarded, but in general they have a favourable prognosis. About $1 / 3^{\text {rd }}$ of low-grade stromal sarcomas recur within 10-15 years; distant metastasis and death from metastatic tumour occur in about $15 \%$ of the cases, but may be long delayed.

Undifferentiated endometrial sarcomas (Formerly high grade stromal sarcomas) bear little histological or antigenic resemblance to the endometrial stroma. These tumours are characterized by high mitotic rate, extreme cytological atypia (Pleomorphic, undifferentiated, rounded to spindled cells with high mitotic index, loss of progesterone receptors and frequent necrosis). They lack the characteristic arborizing vasculature and the areas of hyalinization characteristic of low grade ESS. Undifferentiated sarcomas of the endometrium occur in older women, have a high mortality and poor prognosis, akin to carcinosarcomas. Most resemble the undifferentiated malignant stroma encountered in Carcinosarcomas.

All pure undifferentiated sarcomas of the uterus including the giant cell variety can for convenience be placed in this category. Pure sarcomas containing cells differentiating along recognizable mesenchymal lines are named in a way that reflects their differentiation, i.e. leiomyosarcomas, osteosarcoma, rhabdomyosarcoma and so on. ${ }^{[3,4]}$ Currently, ESS is staged as per the FIGO Staging for ESS (2008) into 4 stages depending on the extent of spread of the tumour. [5]

\section{AIMS AND OBJECTIVES}

To study the Histo-morphological features of ESS. To study the age distribution as compared to other stromal tumours and to evaluate the prognostic factors of ESS.

\section{MATERIALS AND METHODS}

The present study was conducted jointly at the Malabar Cancer Centre (MCC), Thalassery, Kannur District, Kerala and Kannur Medical College (KMC), Kannur, Kerala, over a four-year period between June 2011 and June 2015. The data related to the cases were retrieved from the Hospital Based Cancer Registry at Malabar Cancer Centre. All patients presenting with pre/peri/postmenopausal bleeding were thoroughly investigated by various modalities including Ultrasonogram (USG)/Computerized Tomography (CT)/Magnetic Resonance Imaging (MRI)/curettage, biopsy and HPE as well as ImmuneHisto-Chemical (IHC) methods apart from a detailed clinical examination.

\section{Inclusion Criteria}

All the cases diagnosed as ESS during the study period were included.

\section{Exclusion Criteria}

All endometrial malignancies other than ESS were excluded. All the patients were treated at Malabar Cancer Centre,
Thalassery. After surgical or hormonal treatment/ radiotherapy, the patients were followed up over a variable period of 1-51 months with a median follow-up time of 7 months.

\section{STATISTICAL ANALYSIS}

Data was entered into Microsoft Excel 2010 data sheet and analysed using SPSS version 22 software. Categorical data was represented in the form of frequencies and proportions. Continuous data was represented in the form of mean and standard deviation. Kaplan-Meier survival analysis was done to find out the survival rate of subjects. $\mathrm{P}$ value $<0.05$ was considered as statistically significant.

\section{OBSERVATIONS AND RESULTS}

The 11 patients diagnosed with endometrial stromal sarcomas belonged to the age group 39-65 years with a mean age of 50 years. The presenting complaints included postmenopausal bleeding or menorrhagia in all cases and/or pedal oedema, abdominal pain and abdominal distension in one case each (Table 1).

\begin{tabular}{|c|c|c|}
\hline $\begin{array}{c}\text { SL. } \\
\text { No. }\end{array}$ & $\begin{array}{c}\text { Presenting } \\
\text { Complaints }\end{array}$ & $\begin{array}{c}\text { Number of } \\
\text { Patients }\end{array}$ \\
\hline 1. & $\begin{array}{c}\text { Postmenopausal bleeding and/or } \\
\text { abdominal distension }\end{array}$ & 7 \\
\hline 2. & $\begin{array}{c}\text { Menorrhagia and/or pedal } \\
\text { oedema, abdominal pain }\end{array}$ & 4 \\
\hline \multicolumn{3}{|c|}{$\begin{array}{c}\text { Table 1: Showing the Incidence of } \\
\text { Complaints of Clinical Presentation (n=11) }\end{array}$} \\
\hline
\end{tabular}

Endometrial curettage/biopsy predicted a possibility of ESS in 4 cases, whereas in 2 cases the differential diagnosis included spindle cell sarcoma and endometrioid adenocarcinoma respectively. In the rest of the cases, the findings were inconclusive. USG/CT/MRI revealed bulky uterus suggestive of fibroid in 7 cases, abdomino-pelvic mass suggestive of ESS in 1 case, heterogeneous irregular mass filling the endometrial cavity suggestive of endometrial carcinoma in 2 cases and retroperitoneal mass in 1 case. Nine patients underwent total abdominal hysterectomy, 1 patient underwent subtotal hysterectomy and the extra uterine retroperitoneal ESS was resected with wide surgical margins. Of the 11 cases studied 9 cases shows polypoidal fleshy lesions (81.8\%), 2 cases showed irregular thickening of myometrium (18\%). Myometrial invasion was observed in a total of 10 cases (90.9\%). Microscopy, in all cases, revealed tumour composed of proliferating cells with endometrial stromal differentiation. Necrosis was observed in 3 cases $(27.3 \%)$. Myometrial invasion was seen in all cases except the extra uterine mass, which showed minimal infiltration into the surrounding structures and one tumour which showed a circumscribed nodule. Spiral arterioles surrounded by tumour cells were seen in 9 out of the 11 cases $(81.8 \%)$. The tumour cells were spindle shaped in 9 cases and round/ovoid shaped in two cases. Pleomorphism was mild in 4 cases, moderate in 3 cases and the rest of the cases showed marked pleomorphism (Fig. 1, 2, 3), (Table 2). 


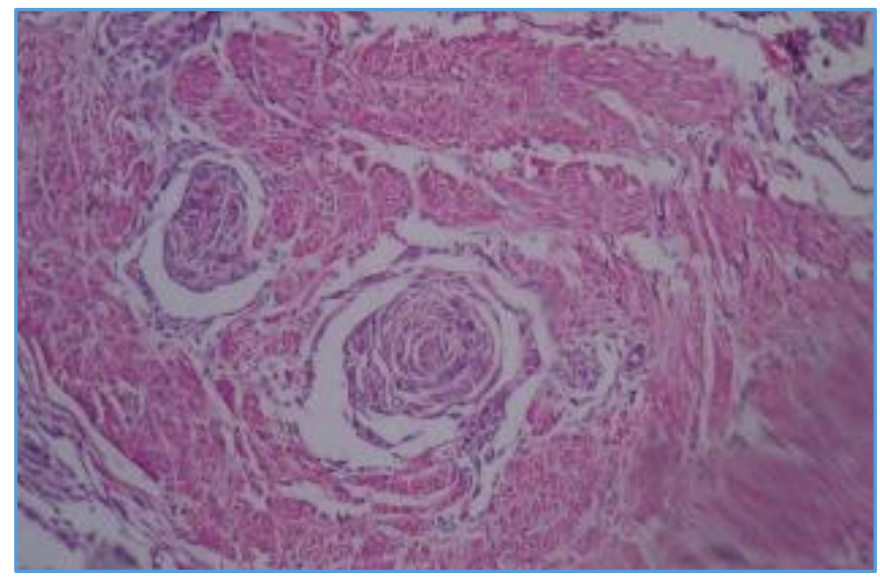

Fig. 1: Showing Low Grade Endometrial Sarcoma with Irregular Tongues of Neoplastic Stroma Invading the Myometrium (H\&E 10X)

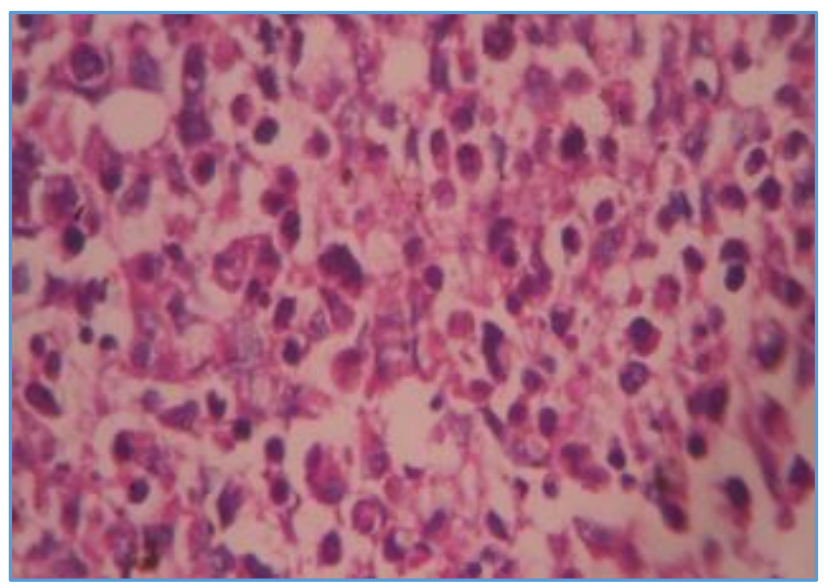

Fig. 2: Showing Undifferentiated Endometrial Sarcoma; Pleomorphic Tumour Cells with Numerous Atypical Mitosis (H\&E 40X)

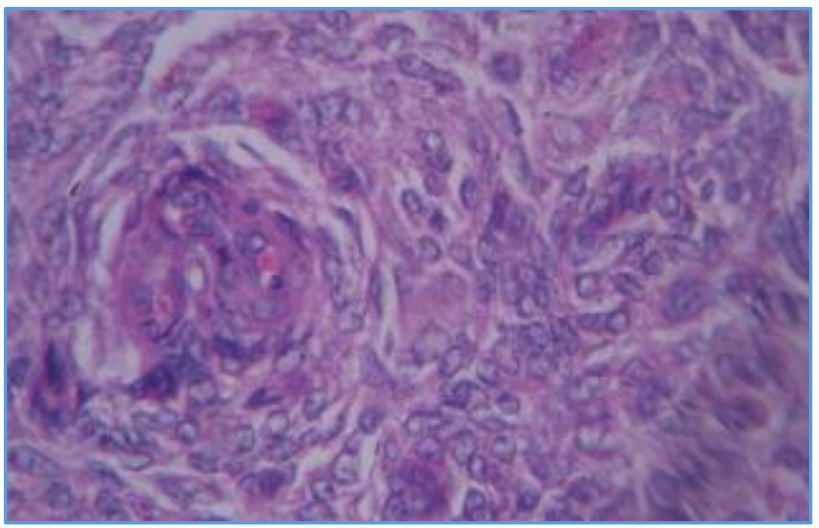

Fig. 3: Low Grade Endometrial Stromal Sarcoma showing Tumour Cells around Spiral Arterioles

\begin{tabular}{|c|c|c|c|c|c|c|c|c|c|c|}
\hline \multicolumn{2}{|c|}{$\begin{array}{c}\text { Cell } \\
\text { Type }\end{array}$} & \multicolumn{3}{|c|}{ Pleomorphism } & \multicolumn{2}{|c|}{ Mitosis } & $\begin{array}{c}\text { Spiral } \\
\text { Arterioles }\end{array}$ & Necrosis & $\begin{array}{l}\text { Lympho- } \\
\text { vascular } \\
\text { Invasion }\end{array}$ & $\begin{array}{c}\text { Epithelioid } \\
\text { Differentiation }\end{array}$ \\
\hline Spindle & Mixed & Mild & Moderate & Marked & $\begin{array}{c}<10 / \\
10 \mathrm{hpf}\end{array}$ & $\begin{array}{c}>10 / \\
10 \mathrm{hpf}\end{array}$ & & & & \\
\hline $72.7 \%$ & $27.3 \%$ & $36.3 \%$ & $27.3 \%$ & $3.3 \%$ & $72.7 \%$ & $27.3 \%$ & $90.9 \%$ & $18 \%$ & $18 \%$ & $18 \%$ \\
\hline
\end{tabular}

Out of 11 cases 6 cases were of low-grade ESS, 4 cases were undifferentiated endometrial sarcomas and one was extra uterine ESS. Radiological evaluation was not diagnostic of ESS in the screened cases. On IHC, 10 cases were found to be CD10 positive (90.9\%). Of the 11 cases, 4 patients were FIGO stage I, 2 patients had stage II disease and 5 patients had stage IV disease. Six patients were administered adjuvant hormonal therapy with or without chemotherapy and radiotherapy, whereas 5 patients were treated with chemotherapy and/or radiotherapy. Seven of the 11 patients showed recurrence of the disease, two patients after 1 month and one patient each after 2, 5, 6, 9 and 51 months. The site of metastasis were lung and para-aortic lymph node in one case; lung, para-aortic lymph node and peritoneum in one case; lung and bone in two cases; lung and cervix in one case; internal iliac vein in one case; and ovary in one case. The mean time interval to recurrence in stage II was 28 months, whereas in stage IV it was 4 months.

The most common site of metastasis was lungs ( 5 out of 7 cases with metastasis). Among 11 cases under follow-up (range 1-51 months) 4 patients, 3 of undifferentiated ESS and
1 of low-grade ESS with lung metastasis expired. The data was analysed using SPSS version 22 software. The overall 4-year survival rate was $62.3 \%$. Survival probabilities were calculated by Kaplan-Meier survival analysis method (Graph 1). $P$ value $<0.05$ was considered as statistically significant. The mean follow-up period was 13.73 months. The recurrence rate was $57.1 \%$ among low-grade tumours and $75 \%$ among undifferentiated tumours. The overall recurrence rate in the present study was $63.6 \%$. The mean duration of survival in low-grade tumour was 44.0+/-6.4 months and in undifferentiated tumours were $3.75+/-1.3$ months. In the study, 4-year survival rate in low-grade tumour was $85.7 \%$ and 6-months survival rate in undifferentiated endometrial sarcoma was $25 \%$. This observation was statistically significant ( $\mathrm{P}$ value of 0.048 ).

Median overall survival was calculated using the Log Rank (Mantel Cox) method, which was statistically significant with a $P$ value of 0.048 . Total mortality rate was $36.4 \%$. The mortality rate with low-grade tumours was $14.3 \%$ and high-grade tumours were $75 \%$. Significant association was observed between FIGO staging and survival of subjects. Out of 4 
subjects in FIGO stage I and 2 subjects in stage II none of them died, whereas out of 5 subjects in stage IV 4 subjects died during the follow-up period. Hence, mortality was observed only in FIGO stage IV. The 4-year survival rate in FIGO stage I and II was $100 \%$, whereas in FIGO stage IV was $20 \%$.

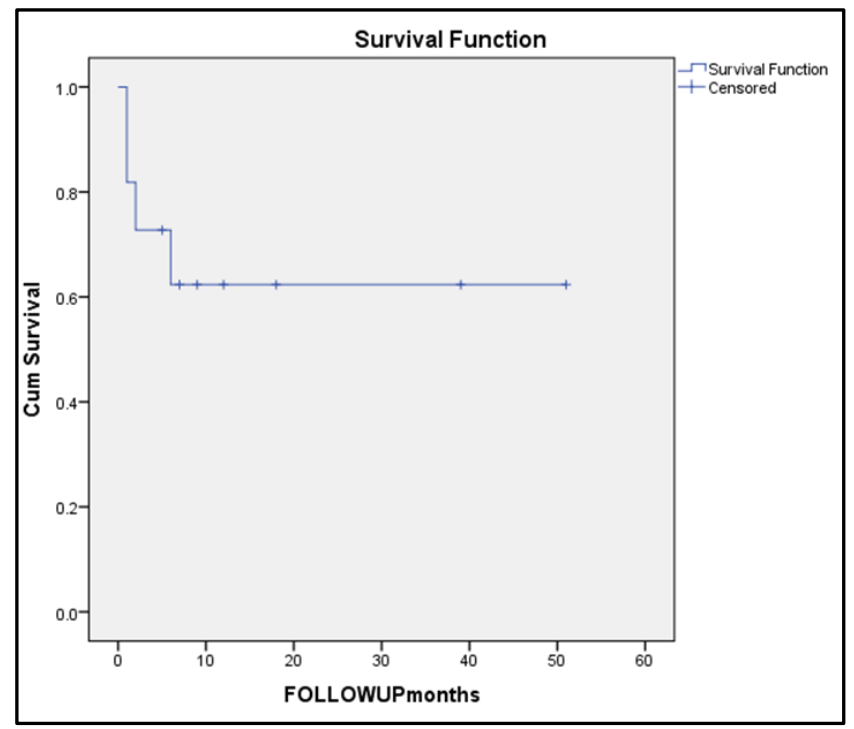

Graph 1: Kaplan Meier Survival Plot showing Overall Survival Rate in Subjects

\section{Immunohistochemistry}

Nine cases were positive for CD 10, one case showed focal positivity for CD 10 and one case was negative. Four cases were positive for ER and 5 cases for PR and the rest of the cases were negative for both ER and PR. Smooth Muscle Actin (SMA) was positive in 2 cases and one case showed focal positivity and in the rest of the cases SMA was negative. Desmin immunoreactivity was negative in all cases (Fig. 4, 5), (Table 3).

\section{IHC Profile of Tumours}

CD10+, 10/11 cases (90.9\%), ER+, 4/11 (36.0\%) PR+, 5/11 cases $(45.45 \%)$; SMA+, (3/11 cases) $(27.3 \%)$.

\begin{tabular}{|c|c|c|c|c|c|}
\hline Case No. & CD10 & ER & PR & SMA & Des min \\
\hline 1. & + & + & + & - & - \\
\hline 2. & + & + & + & - & - \\
\hline 3. & Focal + & - & - & Focal + & - \\
\hline 4. & + & - & + & + & - \\
\hline 5. & + & - & - & - & - \\
\hline 6. & - & - & - & + & - \\
\hline 7. & + & - & - & - & - \\
\hline 8. & + & - & - & - & - \\
\hline 9. & + & + & + & - & - \\
\hline 10. & + & + & + & - & - \\
\hline 11. & + & - & - & - & - \\
\hline \multicolumn{7}{|c|}{ Table 3: Showing IHC Profile of ESS (n=11) } \\
\hline \multicolumn{7}{|c|}{} \\
\hline
\end{tabular}

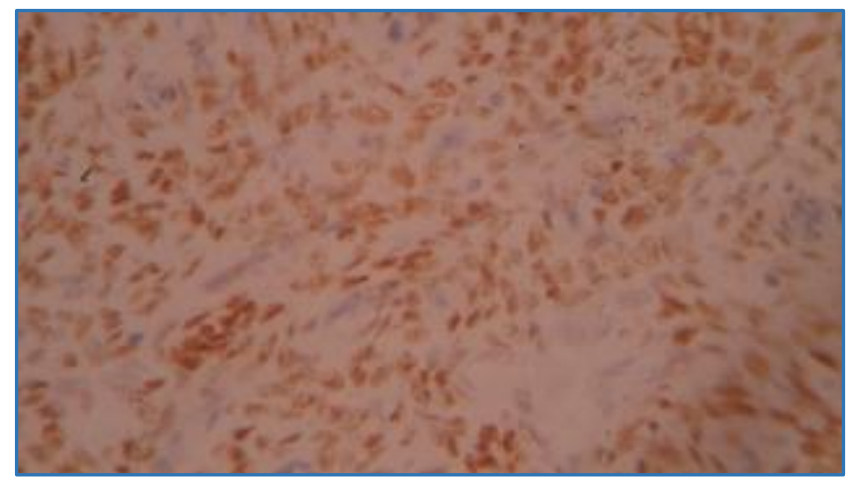

Fig. 4: Showing Low Grade Endometrial Stromal SarcomaCD10 Immunostain; Diffusely Positive Membranous and Cytoplasmic Staining in Stromal Cells (40X)

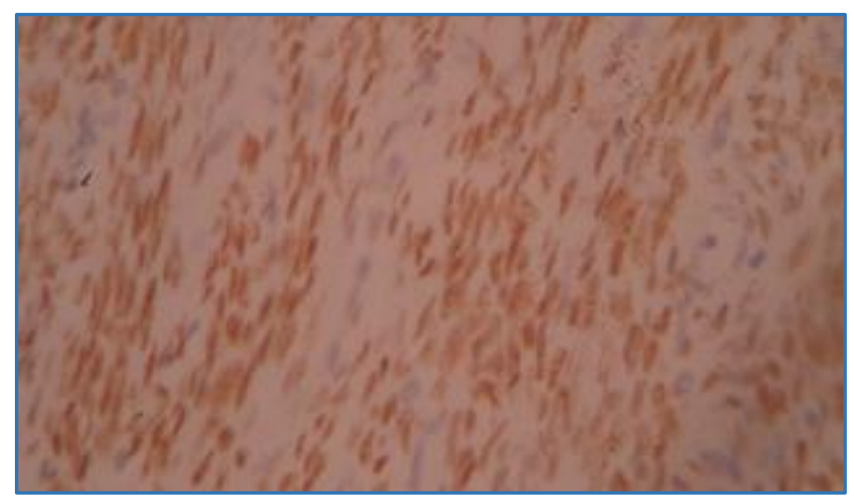

Fig. 5: Showing Low Grade Endometrial Stromal Sarcoma - PR Immunostain - Diffusely Positive Nuclear Staining in Stromal Cells (40X)

\section{DISCUSSION}

Endometrial Stromal Sarcoma (ESS) is a rare gynaecologic malignancy that constitutes $0.2 \%$ of all uterine malignancies and $10-15 \%$ of all uterine sarcomas.[6] The annual incidence is $1-2 /$ million women. [7] In the present study, the patients were investigated for their presenting complaints which included postmenopausal bleeding (63.0\%), menorrhagia (36.0\%) with associated symptoms like pedal oedema (9.0\%), abdominal pain $(9.0 \%)$ and abdominal distension (9.0\%). The mean age of the patients was 50 years, $64 \%$ being postmenopausal. These findings are similar to that found in the study by Puliath G and Nair M K.[2], in which $10-25 \%$ of the patients were premenopausal and the age range of the patients was 42 to 58 years. The concordance rate between clinical and histopathological diagnosis was 36\%.

USG/CT/MRI revealed bulky uterus suggestive of fibroid in 7 cases, abdomino-pelvic mass suggestive of ESS in 1 case, heterogeneous irregular mass filling the endometrial cavity suggestive of endometrial carcinoma in 2 cases and retroperitoneal mass in 1 case. The masses were hyperechoic or isoechoic or anechoic with microcystic areas compared to the normal myometrium, which was similar to the findings in the study by Ga E P and Sung E R et al.[8] In one asymptomatic patient, follow-up sonography showed ovarian mass with hypoechoic and anechoic cystic areas. Grossly, majority of the cases were polypoid fleshy masses with infiltration into the surrounding myometrium. According to the current WHO classification scheme, stromal neoplasms are divided into three categories: 1. Benign endometrial stromal nodules, 2. Low grade ESS.3. Undifferentiated Endometrial Sarcomas.[3] 
Interpretation of an endometrial stromal neoplasm in biopsy or curettage material first depends on its recognition as a neoplastic stromal process and its distinction from potential mimics including endometrial basalis, aglandular functionalis, endometrial polyp and adenosarcoma. Multiple fragments of aglandular cellular stroma containing spiral arteriole like vessels are characteristic of a stromal neoplasm. Distinction from fragments of basalis is made by virtue of the presence of an orderly component of glands in the latter and lack of rich vasculature, so characteristic of stromal neoplasia. Strips of aglandular functionalis usually associated with submucous leiomyomata tend to be less cellular and show features of compression and reactive surface changes. Fragments of stromal endometrial polyps usually exhibit other features of polyp including large thick-walled vessels and abnormal glandular architecture. Adenosarcoma may also exhibit a cellular stroma, but typically have glandular cuffing, although sometimes subtle. Appreciation of a more spindled atypical stroma without a rich vascular network helps facilitate this distinction. Determination between benign and malignant neoplasm is based on whether or not there is myometrial or lymphovascular invasion, two criteria that are difficult to assess in curettage. In general, diagnosis of an endometrial stromal neoplasm with a comment on one's inability to distinguish between a stromal nodule and sarcoma in the submitted material will be the most likely course of action.[9] The differential diagnosis of endometrial stromal tumours includes uterine smooth muscle tumours. Endometrial stromal tumours with smooth muscle differentiation or those that have a more fibrous or myxoid appearance may occasionally be confused with uterine smooth muscle tumours. Conversely, uterine smooth muscle tumours may mimic endometrial stromal tumours, particularly when the former is markedly cellular, e.g. highly cellular leiomyoma or has prominent vascular invasion, e.g. intravenous leiomyomatosis and intravenous leiomyosarcomatosis.[7] In difficult cases, a panel of biomarkers including $\mathrm{H}$-caldesmon, desmin and CD10 may be helpful.[9] Low grade sarcomas may exhibit other forms of differentiation including smooth muscle and sex cord differentiation. In the latter form, the tumour contains epithelial-like or sex cord-like elements often with epithelioid appearance, arranged in nests, cords, trabeculae, solid or tubular structures. If this element predominates, the tumour is considered to be a uterine tumour resembling ovarian sex cord tumour and may cause diagnostic difficulties.[10] In the present study, 7 of the 11 patients (63.6\%) showed recurrence of the disease; 2 patients each after 1 month, one patient each after 2, 5, 6, 9 and 51 months. The median time for recurrence was 5 months. Four patients died of the disease during the period of follow-up. The commonest site of metastasis in the present study was lungs ( 5 out of 7 cases) - (overall 45.5\%) which is similar to that in earlier studies.[8,11] Of the patients 7 showing recurrence, 4 patients had low-grade ESS (Overall 36.3\%) and 3 had undifferentiated endometrial sarcomas (overall 27.3\%) based on the histomorphological features/mitotic counts. The mean time interval to recurrence among low-grade ESS was 16.75 months, whereas among undifferentiated ESS it was 3.75 months. The mean time interval to recurrence in stage II was 28 months, whereas in stage IV it was 4 months.

Late recurrences are common in low-grade ESS, even in early stage disease. In patients with stage I disease, the median time to recurrence is 65 months compared to 9 months in patients with stage III-IV ESS, therefore long-term follow-up is recommended. Recurrences occur in 36-56\% of patients, most frequently in the pelvic cavity or the lungs. Metastatic spread to the cerebrum and bones and invasion in the great vessels have also been described. Recurrences in the abdominal cavity are rare. Recurrences arising in the small bowel and colon are intimately associated with endometriosis as the rectosigmoid has the highest incidence of intestinal endometriosis.[11] In the present study, one case of undifferentiated endometrial sarcoma showed lymphovascular invasion, but was asymptomatic till 7 months of follow-up. None of the cases showed combination of low grade and undifferentiated endometrial sarcoma or arterial embolus.

In cases with a combination of low-grade ESS and undifferentiated component and arterial tumour embolus on histomorphological study, the risk of recurrence is markedly elevated as demonstrated in the study by Feng D and Wolfson D. ${ }^{[12]}$ wherein the patient was a premenopausal young woman with predominantly low-grade ESS admixed with a small component of Undifferentiated Endometrial Sarcoma with Nuclear Uniformity (UES-U). The aggressiveness and survival theoretically would be expected to be between low-grade ESS and Undifferentiated Endometrial Sarcoma with Nuclear Pleomorphism (UES-P). However, the histopathology in this patient demonstrated an arterial tumour embolus along with extensive lymphangio-vascular invasion, which indicated the extremely high risk of rapid recurrence and distant metastasis. Malignant transformation in endometriosis is considered to be an unusual event, only occurring in $0.7-0.1 \%$ of cases.

As reported, most of the malignant tumours that originate from endometriosis are endometrioid adenocarcinoma and clear cell types and endometrial stromal sarcoma is extremely unusual. ESS, which is characterized by cells that resemble those of the endometrial stroma during the proliferative phase, usually originates from the uterine corpus, although it may arise in extra-uterine locations.[13] In the present study, one patient with extra uterine ESS was detected which was retroperitoneal in location. The most common sites of extra uterine ESS are in the pelvis, whereas gastrointestinal involvement is rare.[14] Tanushree $\mathrm{G}$ and Arunava $\mathrm{R}$ et al,[15] reported a case of multiple primary extra uterine ESS in the pelvis and abdomen in association with endometriosis. Similarly, Chang K L and Crabtree G S et al,[16] reported 20 patients with primary extra uterine endometrial stromal sarcoma at various sites including ovaries, fallopian tube, pelvic cavity, abdominal cavity and retroperitoneum.

In both stromal nodules and low-grade endometrial stromal sarcoma, chimeric JAZF1-JJAZ1 mRNA transcripts can be detected by reverse transcriptase Polymerase Chain Reaction (PCR) in the majority of cases. Moreover, FISH analysis performed on Formalin-Fixed Paraffin Embedded (FFPE) tissue show evidence of the $t(7 ; 17)$ translocation in both endometrial stromal nodules and low-grade ESS suggesting that stromal nodule may be a precursor lesion for low-grade ESS. In addition, FISH shows evidence of the translocation in mixed endometrial stromal-smooth muscle tumours in both the endometrial stromal and smooth muscle component, supporting the concept that these tumours are of endometrial stromal derivation.[8] Unfortunately, there are no histopathological parameters in low-grade ESS to predict 
which patients with tumours confined to the uterus are at risk of recurrence.

Distant metastasis principally involving the lung may occasionally occur often close to a decade following initial presentation.[8] The 4-year survival in FIGO stage I and II was $100 \%$ and 9 months survival in FIGO stage IV was 20\%, which is similar to that in the study by Puliath G and Nair K M, which found a 5-year survival rate of $54-100 \%$ at FIGO stage I, 30\% at stage II and only $11 \%$ at FIGO stage III and IV. In yet another similar study by Chan J K and Kawar N M.[17], the 5-year survival of $89.3 \%$ for stage I and II patients and $50.3 \%$ for stage III and IV (which is much higher than in the present study). In the present study 10 out of 11 cases were positive for CD 10, 4 cases were positive for ER, 5 cases were positive for PR, 2 cases were positive for SMA and desmin was negative in all the cases which is similar to the findings by Puliath $G$ and Nair M K.[2] as well as Kim J Y and Hong S Y.[18] The majority of ESS will be CD10 positive (Diffusely)/h-caldesmon negative/desmin variable (But usually focal) and the majority of leiomyosarcomas will be h-caldesmon positive/desmin positive/CD10 variable (Often positive).

The main pitfall to consider are that nearly half of leiomyosarcomas can be CD10 positive (Sometimes diffusely); some ESS are diffusely positive for desmin and some ESS can be CD10 negative. ESS are typically not caldesmon positive unless there are areas of smooth muscle differentiation. In the latter situation, interpretation of areas with classic morphology will facilitate the correct diagnosis. In the distinction with leiomyosarcomas, areas of smooth muscle differentiation in ESS tend to be bland and will not exhibit the degree of cellularity and nuclear pleomorphism that can be present in leiomyosarcomas. ${ }^{[9]}$ Antibodies against CD10 mark a substantial number of hemangiopericytomas and solitary fibrous tumours either focally or in a patchy cytoplasmic and membranous pattern and should always be combined with anti-oestrogen receptor and CD34 when the differential diagnosis includes ESS.[19]

\section{CONCLUSION}

ESS is a rare entity and a diagnostic challenge. In addition to the classical morphological features, immunostaining for CD10 is of high value in diagnosis. ER and PR positivity shows these tumours are amenable to hormonal therapy. As these tumours have tendency for late recurrence long-term followup is essential, i.e. once in 3 months for first year, half yearly for next 4 years and annually thereafter.

\section{ACKNOWLEDGEMENT}

We sincerely thank Dr. Saina and staff of Department of Cancer Registry and Epidemiology, MCC; the Management, Malabar Cancer Centre, Thalassery, and Kannur Medical College, Kannur, for their continuous support and encouragement.

\section{REFERENCES}

1. Sharma DN, Rath GK, Kumar S, et al. Clinical outcome of patients with uterine sarcomas. Journal of Cancer Research and Therapeutics 2011;7(3):270-4.

2. Puliyath G, Nair MK. Endometrial stromal sarcoma: a review of literature. Indian J of Med Paediatr Oncol 2012;33(1):1-6. Available from:

http://www.ijmpo.org/text.asp?2012/33/1/1/96960.
3. George LM, Tan AI. Endometrium. In Diagnostic histopathology of tumours. $3^{\text {rd }}$ Edn, Christopher DM Fletcher Editor. 2007;1:652-71.

4. Michel RH, Teri AL, Richard LK. The uterine corpus. In Sternberg's diagnostic pathology Stacey EM, Darryl C, Joel $\mathrm{K}$, et al. editors; 4th edn. Lippincot Williams and Wilkins. Jaypee Brothers Medical Publisher's 2006;3:2435-542.

5. Prat J. FIGO staging for uterine sarcomas. International J Of Gynaecology and Obstetrics 2009;104(3):177-8.

6. Diesing D, Cordes T, Finas D, et al. Endometrial stromal sarcoma: a retrospective analysis of 11 . Anti cancer Res 2006;26(IB):655-61.

7. Pink D, Lindner T, Mrozek A, et al. Harm or benefit of hormonal treatment in metastatic low-grade endometrial stromal sarcoma: single center experience with 10 cases and review of the literature. Gynaecologic Oncology 2006;101(3):464-9.

8. Ga EP, Sung ER, Soon NO, et al. Ultrasonographic findings of low-grade endometrial stromal sarcoma of the uterus with a focus on cystic degeneration. Ultrasonography 2016;35(2):124-30. Published online Oct 5, 2015.

Available from: http://dx.doi.org/10.14366/usg.15045

9. Marissa RN, Bradley JQ. Uterine mesenchymal tumours. In Diagnostic gynaecologic and obstetric pathology. Christopher PC, Kenneth RL, Editors. Elsevier Publications 2006;611-73.

10. Sutak J, Lazic D, Cullimore JE. Uterine tumour resembling an ovarian sex cord tumour. J Clin Pathol 2005;58(8):888-90.

11. Ilsalien SB, Miriam LH, Freek CP, et al. A very late recurrence of a formerly misdiagnosed low grade endometrial stromal sarcoma metastasized to the colon. Int J Surg Case Rep 2013;4(12):1113-6.

12. Feng D, Wolfson D. Endometrial stromal sarcoma of the uterus with arterial tumour embolus. The Internet Journal of Gynaecology and Obstetrics 2008;12(1).

13. Chunyan L, Xin H, Suxia L, et al. Endometrial stromal sarcoma arising from endometriosis: a clinicopathological study and literature review. Gynaecol Obstet Invest 2012;74(4):288-97. Accessible online at: www.karger.com/goi

14. Andres A, Oluwole F, Dineo K. A case of extrauterine endometrial stromal sarcoma in the colon diagnosed three decades after hysterectomy for benign disease. Case Reports in Obstetrics and Gynaecology Article ID 202458, 2013;3 pages.

Available from: http://dx.doi.org/10.1155/2013/202458.

15. Tanushree G, Arunava R, Susy K. Primary extra uterine endometrial stromal sarcoma: located in pelvic and abdominal tissue and arising in endometriosis. Indian J Pathol Microbiol 2014;57(3):447-9. Available from: http://www.ijpmonline.org/text.asp?2014/57/3/447/ 138757.

16. Chang KL, Crabtree GS, Lim-Tan SK, et al. Primary extra uterine endometrial stromal neoplasms: a clinicopathological study of 20 cases and a review of literature. Int J Gynaecol Pathol 1993;12(4):282-96. 
17. Chan JK, Kawar NM, Shin JY, et al. Endometrial stromal sarcoma-a population based analysis. British Journal of Cancer 2008;99(8):1210-5. Available from: www.bjcancer.com

18. Kim JY, Hong SY, Sung HJ, et al. A case of multiple metastatic endometrial stromal sarcoma, arising from an ovarian endometriotic lesion. J Gynaecol Oncol 2009;20(2):122-5.
19. Rohit B, Jinru S, Amanda JH, et al. Distinction of endometrial stromal sarcomas from 'hemangiopericytomatous' tumours using a panel of immunohistochemical stains. Modern Pathology 2005;18(1):40-7. 